\title{
Immunofluorescence Microscopy As a Critical Confirmation Technique in Cell Cycle-Related Proteomics Studies
}

\author{
Silviu L. Faitar ${ }^{1}$ \\ 1. Department of Mathematics and Natural Sciences, D’Youville College, Buffalo, NY USA.
}

The EVI5 gene was originally identified in a screen for integration sites of ectopic viruses that gave rise to tumors in mice [1]. The human EVI5 gene was isolated and characterized as a result of its location at the breakpoint junction of a $\mathrm{t}(1 ; 10)(\mathrm{p} 21 ; \mathrm{q} 21)$ constitutional chromosome translocation in a patient with stage 4S neuroblastoma [2]. The product of the human EVI5 gene is an 810 amino acids long ubiquitously expressed protein. It carries two readily identifiable motifs [2,3], a Tre2/Bub2/Cdc16 (TBC) consensus sequence domain in the N-terminal region, which is known in other proteins to act as a GTPase activating domain (GAP), and an extensive coiled coil in the C-terminal region, which indicates possible protein-protein interaction sites. A conserved nuclear localization signal is also present in the N-terminal region. Previous immunocytochemical studies revealed that the Evi5 protein has a dynamic intracellular localization during the cell cycle, being associated with the mitotic spindle during metaphase, and migrating to the midzone and midbody in late mitosis and during cytokinesis [3]. Little is known however, about the function of the normal Evi5 protein or its possible association with neuroblastoma pathogenesis. To identify the putative metabolic pathways that involve Evi5, a functional proteomic approach was used in order to determine the identity of proteins that interact with it [4].

The TBC-containing N-terminal part and the coiled-coil rich C-terminal part of Evi5 were expressed as GST-fusion proteins, and GST pull-down experiments were performed using total protein extracts. The proteins that specifically bind to the two Evi5 fusion proteins were analyzed by a linear ion trap mass spectrometer (Thermo Electron LTQ) coupled to a nanochromatography system. A total of 8 proteins were found to interact with Evi5-N and 39 with Evi5-C. They included proteins involved in cytoskeleton reorganization, signal transduction, vesicular transport, membrane traffic and neuronal migration. Among these potential functional partners the highest matching scores were obtained for alpha-tubulin, annexin VI, beta-spectrin, ezrin, and Rab11. The mass spectrometry results were confirmed by western blotting and immunoprecipitation. However, these in vitro techniques do not demonstrate that these proteins actually interact in vivo. Immunoflorescence microscopy studies were necessary to confirm the co-localization of these proteins with Evi5. Furthermore, taking into account the dynamic localization of Evi5 during mitosis and cytokinesis, it is important to determine the specific phase of the cell cycle when these interactions may occur. This study used immonocytochemical stainings and immunoflorescence microscopy to test for these putative functional interactions.

Human epithelial cells (Hep-2) immobilized on glass slides (Bio-Rad-Kallestad) were stained for Evi5, alpha-tubulin, annexinVI and Rab11, using commercially available antibodies. An antibody specific for Rab23 was also used as a negative control for the Evi5-Rab11 co-localization studies, knowing that in spite of the high homology between Rab11 and Rab23, the latter does not interact with Evi5 [4]. DyLight 594-conjugated AffiniPure goat anti-mouse IgG $(\mathrm{H}+\mathrm{L})$ and DyLight 488-conjugated AffiniPure donkey anti-rabbit IgG $(\mathrm{H}+\mathrm{L})$ (both Jackson ImmunoResearch) were used as secondary antibodies. Vectashield mounting medium with DAPI (Vector Laboratories Inc.) was used after staining, and the cells were examined using a Zeiss Axioskop 40 fluorescence microscope (Carl Zeiss Microscopy). Images were obtained using a 100 X Zeiss A-Plan oil immersion objective and Immersol 
518F immersion oil. Image acquisition and processing was executed using the ZEN Imaging Software (Carl Zeiss Microscopy).

Immunoflorescence microscopy experiments on interphase cells confirmed a strong Evi5 nuclear staining, as well as a relatively specific cytoplasmic staining that was largely localized to the centrosome. In double-staining experiments, Evi5 showed co-localization with alpha-tubulin at the centrosome, and co-localization with annexin VI in the nucleus (Fig.1). No significant co-localization was observed with either Rab11 or Rab23 in interphase. Both these proteins showed a punctuated cytoplasmic localization consistent with their role in vesicular transport and membrane trafficking (Fig.1). In mitotic cells, there was no observed co-localization between Evi5 and annexin VI. However, as the centrosome divides and forms the polar bodies, Evi5 is located at the spindle poles and colocalizes with the mitotic spindle tubulin (Fig.1). As telophase develops, Evi5 appears on the midzone between the two forming daughter cells and persists at the midbody to the terminal stages of cytokinesis, also showing co-localization with alpha-tubulin. Significantly, a strong Evi5-Rab11 co-localization is observed during the late stages of mitosis and especially during cytokinesis. This dynamic immunofluorescence pattern is not observed when anti-Rab23 antibodies were used instead of antiRab11 antibodies, suggesting a specific functional interaction between Evi5 and Rab11 during the furrow ingression and the vesicular transport associated with cytokinesis.

The microscopy studies presented demonstrate the importance of immunoflorescence as a critical confirmation technique for protein-protein interaction proteomics data, especially when transitory shortterm interactions are investigated. Microscopy images could not only determine if the studied protein are localized in close vicinity to each other, but they also offer a timeline for these interactions, especially in relationship to the cell cycle.

\section{References:}

[1] X Liao, A Buchberg, N and N Copeland, J. Virol. 69, (1995) p. 7132.

[2] T Roberts, O Chernova and J Cowell, Cancer Genet. Cytogenet. 100, (1998) p. 10.

[3] S Faitar, K Sossey-Alaoui, T Ranalli and J Cowell, Experimental Cell Research 312, (2006) p. 2325.

[4] J Dabbeekeh, S Faitar, C Dufresne and J Cowell, Oncogene 26, (2007) p. 2804.
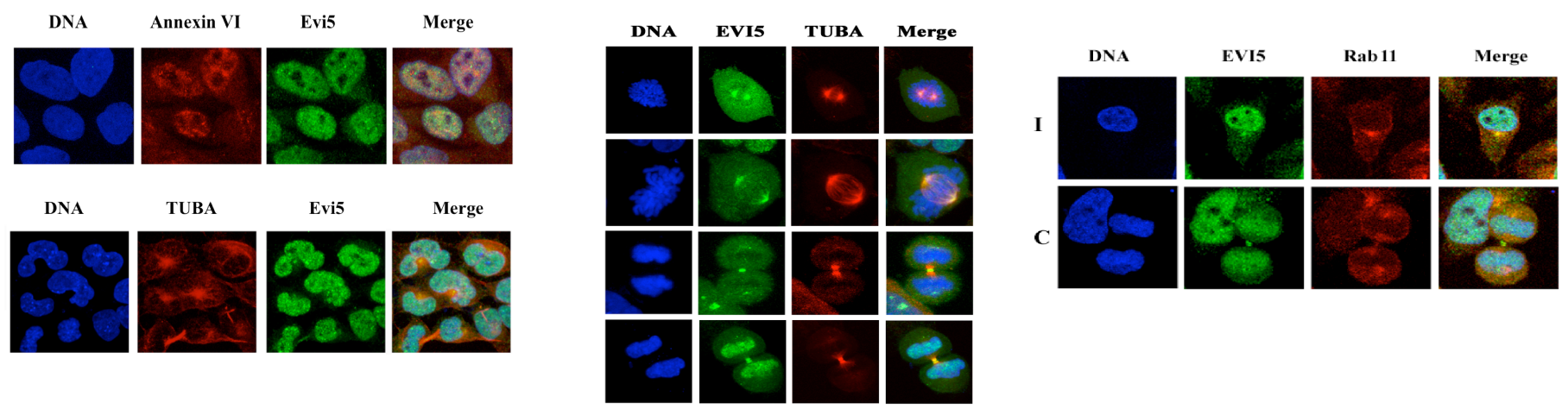

Figure 1. Immunofluorescence microscopy images showing co-localization of Evi5 (green) with annexin VI, alpha-tubulin (TUBA) and Rab11 (red). DNA is stained with DAPI (blue). Hep-2 cells are shown in interphase (left panel) and in various stages of cell division (center and right panels). 